\title{
Understanding Customer Voice of Project Portfolio Management Software
}

\author{
Maruthi Rohit Ayyagari ${ }^{1}$ \\ College of Business, University of Dallas \\ Irving, Texas, USA
}

\author{
Issa Atoum ${ }^{2}$ \\ Department of Software Engineering \\ The World Islamic Sciences and Education, Jordan
}

\begin{abstract}
Project Portfolio Management (PPM) has gained success in many projects due to its large number of features that covers effective scheduling, risk management, collaboration, and third-party software integrations to mention a few. A broad range of PPM software is available; however, it is essential to select the PPM with minimum usage issues over time. While many companies use surveys and market research to get users feedback, the PPM product software reviews carry the voice of users; the positive and negative sentiments of the PPM software reviews. This paper collected 4,775 reviews of ten PPM software from Capttera.com. Our approach has these phases- text preprocessing, sentiment analysis, summarization, and categorizations. The software reviews are filtered and cleaned, then negative sentiments of user reviews are summarized into a set of factors that identify issues of adopted PPM software. We report the most important issues of PPM software which were related to missing technological features and lack of training. Results using Latent Dirichlet Allocation (LDA) model showed that the top ten common issues are related to software complexity and lack of required features.
\end{abstract}

Keywords-Project Portfolio Management (PPM); software reviews; sentiment analytics; text summarization; $L D A$

\section{INTRODUCTION}

Every organization strive to achieve its strategic goals by executing a set of cornerstone projects[1]. Managing and controlling diverse, interrelated projects as a portfolio is nontrivial. The projects face problems related to change management, scoping [2], complexity [3], timelines, and tracking. Project and portfolio managers must harvest the features of project portfolio management (PPM) software to ensure proper control. Common issues of PPM include prioritization [4], inaccurate reporting, resource utilization, and software development lifecycle. The primary objective of PPM is to execute projects that support strategic organization goals under constraints of scope, time and resources.

Recently, an increase in PPM software is noted. According to Gartner [5], the estimated $\$ 2.5$ billion project portfolio management market demonstrates stability, as well as an increasing level of change. The driving forces to PPM software tools are traced back to requirements of PPM practitioners and stakeholders, organization configuration management, demand of collaboration between users, and increased the complexity of enterprise projects [3]. If the PPM software tools are implemented according to business needs, they have the potential to improve organizational business benefits aligned with business strategy at the portfolio level.
The organizations that use PPM tools are $44 \%$ more likely to complete projects on budget, and $52 \%$ more likely to get the anticipated Return on Investment [6]. Implementors and adopters of PPM got a decrease of failure by $59 \%$, spent $37 \%$ less per project, reduced the wide variety of redundant projects by $78 \%$, and increased resource constructiveness by $14 \%$ [7].

Although PPM tools implementation is recognized in practice, current understanding issues of PPM tools are limited [8], [9]. As organizations strive to turn out to be globally aggressive while increasing shareholder's value, they are always compelled to reduce infrastructure costs to get products into the market cheaper, quicker and with better high-quality. Therefore, the PPM software vendors attempt to continue software evolution based on user needs. While users' feedbacks can be gained by surveys[8] where target PPM software is showing an increase in the market place, globalization causes extremely high competition between PPM vendors. Moreover, enterprise software adopts the roles of different levels of practitioners; therefore, studying all these stakeholders could increase vendors' revenue by planning for the next product release. Therefore, the need for a systematic study on the issues behind the diffusion of PPM software tools in organizations is decisive.

This paper develops a taxonomy of PPM software tools issues of a set of selected of 10 favorite PPM software toolsMicrosoft Project, Wrike, Atlassian, Basecamp, Trello, Asana, teamwork projects, Podio, Smartsheet, JIRA. The proposed approach shown in Fig. 1 is based on software reviews of the selected PPM software. We collected 4,775 PPM reviews and yielded 4,397 reviews after removing empty and invalid details. First, we do a preprocessing step by removing stop words and changing words to lowercase. Then, we do sentiment analysis of the cons part of the reviews, as cons are supposed to have negative comments - the output of the sentiment analysis which is the list of negative reviews. Next, we do a summarization based on genism TextRank algorithm [10]. Finally, we allow two coders to read the summarized reviews and analyze potential issues manually. The coders end up with categories and subcategories of PPM software related issues.

A key difference in this paper compared to previous work, is the application of text mining techniques [11] to reduce the overwhelming number of software reviews. Instead of depending of market research that might not give instant output of the PPM issues trend, the proposed approach provides instant results of key success/failure factors of PPM. 


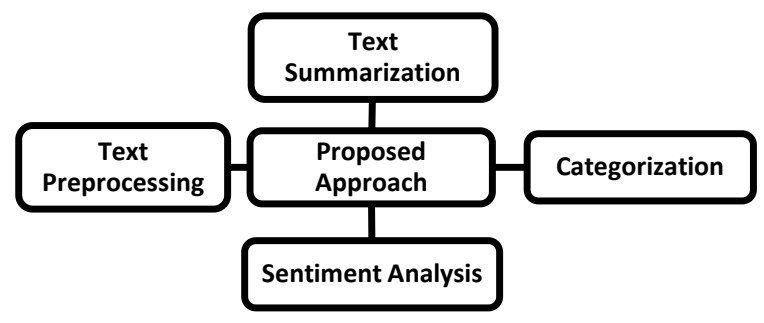

Fig. 1. Context Diagram of the Proposed Model.

There have been many studies in the era of PPM success factors [12]; however, they are limited to identify failure factors of PPM tools [13]. The objective of this paper is to identify the negative voice of the PPM software practitioners so that software vendors can enhance their products accordingly. Therefore, the major contribution here is to both vendors and users. Users have the trend and of PPM software and vendors get mined categorized and instant results of the customers' voice.

The remainder of the paper is structured as follows. Section two summarizes a background of PPM and its issues, and mining software reviews. Section three summarizes related work. Section four illustrates the proposed model while Section 5 evaluates the proposed model. Section 6 provides conclusions, with implications and future research.

\section{BACKGROUND}

\section{A. Project Portfolio Management Software}

The complexity of nowadays enterprise projects makes project management success challengeable for many companies. Many studies showed that poor project management means projects finish late, over-budget, and with the lower return of investment. Therefore, businesses often rely on project management software, which controls the project, manages change, and mitigates risk by identifying potential project issues.

A study from Forrester Research Group concluded that implementing a PPM solution produced an expected ROI of $255 \%$ [14]. The Aberdeen Group, organizations that the use a PPM tool, are 44 percent more likely to complete projects on time [6]. Consequently, an acceptable PPM software should provide features that draw project success with maximum benefits.

Project management software can help streamline business processes by applying state of the art PPM software development frameworks [15]. The process gets increased in difficulty as the number of projects increase. The problem will be how to maximize the success of various interleaved projects given restrictions on time, budget, and resources. Therefore, PPM software gives a hit of decision making by providing visibility, and oversight with dashboard tools to prioritize and manage current projects.

The PPM software tools provide tools to manage and control projects, provide proper communication, collaboration with resources, progress and management reports, risk management, integration with other enterprise application such HR and CRM, and predicting project future.

\section{B. Issues of PPM}

Enterprise PPM software requires extensive configuration to support business goals. The PPM software tools especially those installed and maintained by the organization are difficult to set up due to the complexity of software that needs a large scale set of experience of hardware and software. The next issue that comes in is the learning curve that can take time for practitioners to understand and find needed information [16]. Generally, a project or portfolio manager must take training on specific software to control and monitor gain the maximum from the PPM tool.

As projects are temporary endeavor undertaken to create a unique product or service [17], they are bound to the environment and change management complexities; therefore, customizing the PPM tool to a specific environment, project, size, and user needs and constraints are essential. Moreover, the PPM tool should be easy to use and user-friendly, where practitioners can easily do the job they are seeking.

PPM tools that run on the cloud suffer from poor efficiency and low availability factors that could affect running critical projects [18]. Moreover, without high-level dashboard decision makers could not provide a creative solution [19]. Therefore, most PPM tools provide reporting, dashboards, and integration with enterprise applications.

\section{Mining Software Reviews}

One value-powerful technique to unveil the underlying elements influencing PPM adoption is to research the online software evaluations, furnished with the aid of practitioners on their reviews and practices of PPM systems. Software reviews are an essential source of information for evaluating as it captures the essence of the user's voice. Therefore, the emergent of several software reviews sites has gained attention to may researchers who are most often referred to as option mining or sentiment analysis.

The sentiment analysis is the technique of computationally figuring out and categorizing reviews expressed in a bit of text, mainly to determine whether the author's mindset towards a specific topic, product, and many others are fantastic, sad, or impartial. The valuable information contained in software reviews is critical for users and decision makers.

However, the immense volume of online reviews makes it difficult for users to comprehend text. The Worldwide data will grow $61 \%$ to 175 zettabytes by 2025 according to IDC [20]. Most often software reviews are accompanied with software rating which is a number that shows the user satisfaction of a product. However, the details of user satisfaction factors are latent in the text. Therefore, text mining could reveal hidden success factors or joint issues.

Text mining provides a set of tasks that can be used to mine interesting patterns of data. Sentiment analysis of PPM software reviews enables users to identify the pros and cons of products. The massive amount of text could be reduced using text summarization techniques. Textual content summarization refers to the technique of shortening lengthy pieces of textual content. The goal is to create a coherent and fluent precis having only the main points mentioned within the text. In our 
paper, summarization is based on ranks of text sentences using a variation of the TextRank algorithm [10].

\section{RELATED WORK}

There have been many works that study project portfolio management systems. However, most of them are propriety without details of how results are systematically generated [21]. The problem is that ranking PPM software is done by marketing research leaving the details of why there is a region for improvement on PPM software.

Gartner, a leading research and advisory company provides reports of PPM software tools over the years. According to Gartner, the tools are categorized into four groups known as the magic quadrant group- leaders, challengers, visionaries, niche players. However, the criteria for the Magic Quadrant is leaned towards large vendors than towards buyers [8]. Similarity, Softwareadvice.com, capttera.com provides tools to compare PPM software tools.

Many researches identify reasons for project failures. Failures are due to a list of issues related to organization, people, technical or the process [13]. Failures are often related to lack of executive support, project management skills, communication and risk management. However, these issues are not related to PPM tools. Therefore, without evidence, we cannot adopt or leave a PPM software tool.

The work of [9] proposes a mathematical model prioritize projects to gain maximum resource utilization. Several other works provide best practice techniques to gain project success [22][23]. A review by [24] showed an increased focus on human actors, project management alternative acceptance, adapt to change. The work uses a fuzzy approach to order key success factors of projects [4].

\section{PROPOSED APPROACH}

The PPM Software reviews provide essential feedback about the products usage by practitioners. However, the number of available reviews makes the decision uncomprehensive as a user must read long reviews which are the time-consuming process. Although reviews provide a rating of the product (usually between 1 and 5), the rating does not provide information about why the user was happy or satisfied. Therefore, reviews must be cleaned and then analyzed for possible exciting patterns.

Fig. 2 depicts the context diagram of the proposed approach. The data collection process comprises a set of steps to accumulate software reviews from the website. We choose to scrape PPM reviews from Capterra.com, as the website has a long list of high-quality reviews. The selected PPM software tools are the most rated and used PPM products-Microsoft Project, Wrike, Atlassian, Basecamp, Trello, Asana, Team work Projects, Podio, Smartsheet, JIRA. Our target was to scrape the most recent 500 reviews from each PPM software. We collected 4,775 reviews as Podio had 225 reviews during project execution (step.1). as we were concentrating on the negative sentiment of PPM reviews- which is the cons element in a review- all reviews that have empty cons or invalid details were removed (step 2). Therefore, the total numbers of valid reviews were 4,732 reviews.

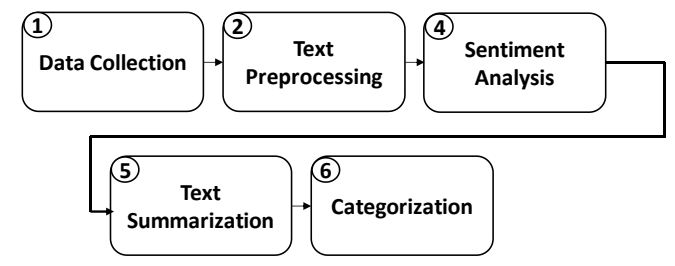

Fig. 2. Proposed Approach.

In the text preprocessing step (step 3), the applied transformations on cons element of the reviews are lowercase, remove accent, parse HTML, remove URLs. Then the resulting text was tokenized using word tokenize pattern next; the text was filtered by removing stop words and all special characters. Consequently, we apply the sentiment analysis algorithm of [25] to identify the negative reviews in the cons element of the reviews. As a result, 63 reviews were omitted from the reviews resulted in 4,669 reviews (step 4).

To reduce the number of reviews, we applied a summarization implementation of Gensim [26] that is based on TextRank Algorithm [10], one of the summarization algorithms that output useful results (step 5). The summarization reduced reviews by $95 \%$ (234 reviews).

The last step (step 6) was completed by two project management experts to ensure consistency and validity. The resultant reviews (cons element) along with other details were passed to the experts, and they were asked to look at the cons part and identify the issue of the reviews. They did the job alone in the first phase, and they could discuss and find a solution when disagreements were found. Before they commenced, we discussed with them and described to them that the objective of their job; the objective to see the failure factors that make the PPM software practitioners unhappy with the software.

\section{EVALUATION AND DiscUSSION}

\section{A. Validating Proposed Approach with Experts}

The main reported issues of the PPM software where categorized by experts as shown in Table I, ordered based on how issues are mostly covered by reviews (frequency). The most common issue that practitioners face is the complexity of the PPM tools. To support large scale projects vendors had added many features to the software that added extra complexity to users; they had difficulties in finding information, managing cards, set permission, and manage complex projects. Therefore, the finding draws attention to vendors that they should find a way to configure the tool as per project size by probably hiding advanced features unless requested. A few junior practitioners reported that the used language in the PPM interface was not easy to understand; mainly the help material was comprehensive but not will be categorized in many cases. An overall view of the word is shown in Fig. 3. According to the figure, users are worried about time, task, use, features as shown in the middle of the figure.

Furthermore, we discovered that the PPM suffers from issues related to ease of use. Because studied PPM software is large-scale software, the users reported that the software was 
not easy to use which is an indirect result of software complexity. They reported that they were not able to navigate quickly and some software was lacking needed project templates for perceived ease of use. This finding direct vendors to customize the navigation and reduce options per needs, perhaps by project size or based on user experience. Given a complicated PPM software that is not always userfriendly, the learning curve was increased as reported by users.

There were issues related to the unavailability of needed features. Many users reported that there were not able to do require reporting unless they integrate with other third-party tools. They also reported that the most common missing feature was handling risk management as part of the PPM software. Therefore, vendors could take this missing feature in the upcoming versions of the software.

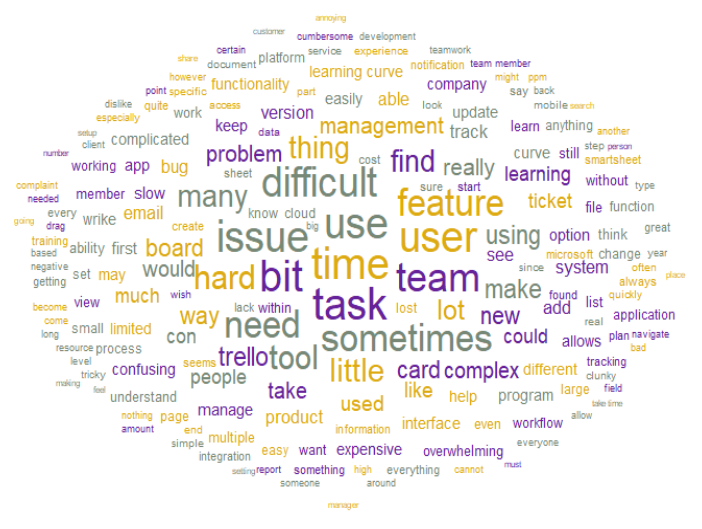

Fig. 3. Word Cloud of PPM Software Issues.
Furthermore, the PPM software for critical applications needs a cloud system that is available and efficient in situations where data is exchanged in complex projects. However, non-cloud-based PPM tools need extended configuration and initial setup before startup.

Also, one common suggestion by users is that the PPM tool should support the automation of some tasks-workflows and triggering task completions automatically.

\section{B. Validating Proposed Approach with Topic Modeling}

We are aware of other related works that discuss the issues of PPM software adoption[12], however, most of studied works have different comparison parameters [13] that does not map with our approach. Moreover, the intent of this research is the negative voice of the customer not the technique used to get this voice; therefore, we opt out comparisons with market researchers or comparing datamining techniques performance. Therefore, another additional technique was used to validate the proposed model.

We run another experiment to show that the summarization algorithm does not omit the necessary PPM issues. We run topic modeling, the latent Dirichlet allocation (LDA) of [27], [28], an unsupervised method that can identify top topics discussed by users. The LDA results of the set of reviews are shown in Table II. Using the terms of the topic models, we assign the topic relative to our findings in column (3). However, we were not able to map the last two topics, due to the accuracy and completeness of the summarization carried out on cons element of the review only. Consequently, our previous findings are $80 \%$ accurate compared to topic modeling; therefore, our findings are complete and more accurate than automated topic modeling results.

TABLE I. TAXONOMY OF PPM SOFTWARE ISSUES (NOTE THAT REVIEWS HAVE SPELLING ERRORS, WE KEEP THEM AS IS)

\begin{tabular}{|c|c|c|}
\hline Issue Category & Issue Subcategory & Sample PPM Review \\
\hline \multirow{13}{*}{ Complexity } & resource card management & \multirow{13}{*}{$\begin{array}{l}\text { Given the robust features and functions that Jira supports, } \\
\text { there's a lot of depth and breadth to the software, so it can be } \\
\text { somewhat complex and confusing to newer users, especially } \\
\text { those who haven't worked much with project management tools } \\
\text { previously. For project managers who are leading multiple } \\
\text { teams simultaneously, things can get a bit overwhelming } \\
\text { because the email notifications and project alerts can quickly } \\
\text { start to become excessive, and there's no way to consolidate } \\
\text { notifications across projects/teams. }\end{array}$} \\
\hline & too many data update & \\
\hline & extra plugins and options & \\
\hline & task management: task for managers, filter tasks, dependency, and change & \\
\hline & manage complex projects & \\
\hline & setting permissions & \\
\hline & match project size & \\
\hline & tracking complexity & \\
\hline & plan execution & \\
\hline & control resources & \\
\hline & setup addon & \\
\hline & Comprehensive help material & \\
\hline & difficult interface language & \\
\hline \multirow[b]{4}{*}{ Ease of use } & templates & \multirow{4}{*}{$\begin{array}{l}\text { This isn't really a con, but there is a bit of a learning curve in } \\
\text { terms of navigating and understanding how to utilize all of the } \\
\text { features. Navigation is not completely innate so that took a } \\
\text { minute to figure out. It's a big tool though so that shouldn't be a } \\
\text { surprise. They have great resources and support to help new } \\
\text { users get adjusted, which is awesome. It only took a day or so of } \\
\text { messing around with the different features to feel fully } \\
\text { comfortable in the capacity that I needed. }\end{array}$} \\
\hline & navigation & \\
\hline & User experience (UX) & \\
\hline & find information & \\
\hline Learnability & - & $\begin{array}{l}\text { There's a very steep learning curve. I'm writing from the } \\
\text { perspective of a product manager so it could be that developers }\end{array}$ \\
\hline
\end{tabular}




\begin{tabular}{|c|c|c|}
\hline & & $\begin{array}{l}\text { have a much easier time grasping the concepts here. For me, it } \\
\text { took awhile to get an understanding of the relationships for } \\
\text { setting up the sprint logic and mapping the progression of a } \\
\text { story through the board. Once I started to immerse myself in it, I } \\
\text { started to grasp it more and more. That said, I could see a more } \\
\text { in-depth sample project being incredibly helpful for tutorializing } \\
\text { within the tools themselves. }\end{array}$ \\
\hline \multirow{6}{*}{ Lack of features } & built in reports & \multirow{6}{*}{$\begin{array}{l}\text { This software does not have some of the features like time sheets } \\
\text { and a risk management section to help tract risks and keep } \\
\text { everyone up to speed with risks. } \\
\text { We trialed Microsoft PPM in one of our subsidiaries, which is a } \\
\text { multi-platform/mixed operating system entity. Microsoft PPM } \\
\text { caused several issues for our workflows. }\end{array}$} \\
\hline & risk management & \\
\hline & manage old tickets & \\
\hline & export file types & \\
\hline & comparative's features & \\
\hline & type of addon & \\
\hline \multirow{4}{*}{ Customization } & workflow customization & \multirow{4}{*}{$\begin{array}{l}\text { Even if TRELLO have great options and features, the } \\
\text { customization of the screen is a little tricky, I wish to reorder } \\
\text { some social alerts on my own hierarchy and the most time } \\
\text { TRELLO over my desires and rearrange everything and it's } \\
\text { really frustrating to rearrange everything each time that I login } \\
\text { to my account. }\end{array}$} \\
\hline & admin involvement & \\
\hline & lack of boards & \\
\hline & edit board & \\
\hline \multirow[b]{3}{*}{ Configuration } & software setup required & \multirow{3}{*}{$\begin{array}{l}\text { When we scaled, the License was not as flexible in higher user } \\
\text { tiers as it was in the lower ones. Also once upon a time an } \\
\text { update went wrong and we had to invest a bucketload of time } \\
\text { and brain power to get everything back up to speed. We did run } \\
\text { jira on premise and hat some custom plugins so there might be } \\
\text { some of the tripwires: In case you use plugins, they must be } \\
\text { available in the version you wish to upgrade to and this might } \\
\text { require some research prior to updates. Also, you can make a } \\
\text { science out of the workflow and notification configuration. In } \\
\text { case it is not documented well when you do it it might get you } \\
\text { into trouble. }\end{array}$} \\
\hline & configuring PPM software & \\
\hline & integration & \\
\hline \multirow{4}{*}{ Collaboration } & log meetings & \multirow{4}{*}{$\begin{array}{l}\text { I have been using this software for the last year and I still can't } \\
\text { find a way to assign a task to more than one person. This seems } \\
\text { like a great software if each project is solely owned by one } \\
\text { person. The collaboration with this program is not the greatest, } \\
\text { but for individual projects it is great. }\end{array}$} \\
\hline & overwhelming emails & \\
\hline & allow user-user communication & \\
\hline & log surveys & \\
\hline \multirow[b]{2}{*}{ Service Quality } & efficiency & \multirow{2}{*}{$\begin{array}{l}\text { Because it is a web application, if you present problems with the } \\
\text { internet connection you will not be able to access the } \\
\text { information of your project, it is always a negative point since } \\
\text { the data should be available. }\end{array}$} \\
\hline & availability & \\
\hline \multirow{4}{*}{ Automation } & drag and drop & \multirow{4}{*}{$\begin{array}{l}\text { The product requires a high investment of time to not only } \\
\text { understand yourself but also train your team, when I became a } \\
\text { mid-level user I found it tedious to set up projects in the } \\
\text { customizable way we required. }\end{array}$} \\
\hline & automate workflows & \\
\hline & trigger cards & \\
\hline & reminders & \\
\hline \multirow[b]{2}{*}{ General } & cross-technology compatibility & \multirow{2}{*}{$\begin{array}{l}\text { There have been times when I've thought that the lingo, they use } \\
\text { on the app might be confusing for new users, or that other } \\
\text { wording would have better explained what a certain tool was. }\end{array}$} \\
\hline & $\cos t$ & \\
\hline
\end{tabular}

TABLE II. TOP 10 TOPICS OF ISSUES OF PPM SOFTWARE TOOLS

\begin{tabular}{|l|l|l|}
\hline$\#$ & Terms & Topic \\
\hline 1 & tool, found, option, small, integration, interface, isn, cost, due, improved & complexity \\
\hline 2 & features, bit, app, people, difficult, confusing, love, platform, understand, slow & lack of features \\
\hline 3 & software, users, learning, management, issues, user, good, nice, support, team & learnability \\
\hline 4 & setup, tickets, button, ticket, navigate, connection, awesome, screen, bugs, phone & configuration \\
\hline 5 & make, platform, email, visually, back, navigate, loved, moving, function, order & ease of use \\
\hline 6 & management, create, add, information, made, nice, update, items, fast, drop & service quality \\
\hline 7 & support, reporting, tools, easier, integrations, workflows, customer, number, files, unlike & configuration \\
\hline 8 & tool, sheet, support, collaboration, dislike, samepage, share, high, move, task & collaboration \\
\hline 9 & time, complex, drag, ability, manage, found, smartsheet, file, program, personally & mixed \\
\hline 10 & work, create, view, clunky, excel, bit tricky, apps, flow, ons, products & mixed \\
\hline
\end{tabular}




\section{IMPLICATIONS}

The implication of this work affects the PPM software vendors who should find the current issues that are degrading their software. Therefore, they could plan their maintenance activities better to fix bugs available bugs. Moreover, the results show that vendors should take care more of user needs of usability and User Experience (UX) issues. Furthermore, they should change their training and software design strategies to reduce software complexity. On the other hand, users could get a glance of what they could choose based on previous analyzed knowledge and experience of previous practitioners.

\section{LIMITATIONS}

Although the proposed approach has reported interesting issues of PPM software readers has to consider a few limitations. The list of reviews was collected from one site; therefore, researchers should consider this before generalizing results. Also, we assume that the latest 500 reviews have the issues of the current version of the PPM; however, it might always be right all the time especially in cases where the practitioner is admired by the software. Moreover, all the ten most popular PPM software is included in as coherent contiguous documents, which limits the identity of the PPM that raises the issue.

\section{CONCLUSION}

This paper proposes an approach to identify the most common issues of PPM software. The approach is based on sentiment analysis to choose negative reviews and on summarization techniques to reduce the number of reviews. The study was carried out over ten popular PPM softwareMicrosoft Project, Wrike, Atlassian, Basecamp, Trello, Asana, teamwork projects, Podio, Smartsheet, and JIRA. We reported the issues fine-grained with the issue category. Results reported showed that the issues are related to complexity, ease of use, learnability, lack of features, customization, collaboration, service quality, and configuration. Our findings reported that users were not worried about compatibility and cost of PPM software. In the future, we will show issues per PPM software.

\section{REFERENCES}

[1] O. Zwikael, Y.-Y. Chih, and J. R. Meredith, "Project benefit management: Setting effective target benefits," Int. J. Proj. Manag., vol. 36, no. 4, pp. 650-658, 2018.

[2] S. Shafiee, K. Kristjansdottir, L. Hvam, and C. Forza, "How to scope configuration projects and manage the knowledge they require," J. Knowl. Manag., vol. 22, no. 5, pp. 982-1014, 2018.

[3] W. Vogel and R. Lasch, "Single approaches for complexity management in product development: An empirical research," in Supply Management Research, Springer, 2019, pp. 151-215.

[4] T. Yaghoobi, "Prioritizing key success factors of software projects using fuzzy AHP,” J. Softw. Evol. Process, vol. 30, no. 1, p. e1891, 2018.
[5] D. B. Stang and M. Light, "Gartner, Magic Quadrant for Project Portfolio Management, Worldwide," 2018.

[6] The Aberdeen Group, "Managing the Project Portfolio to Improve Profits," 2014.

[7] IDC, "Measuring the ROI of PPM (Project and Portfolio Management)," 2018.

[8] T. Byrne, "Looking beyond the magic quadrant to find the nitty-gritty," 2009. [Online]. Available: https://www.realstorygroup.com/Blog/1660Looking-beyond-the-magic-quadrant-to-find-the-nittygritty.

[9] B. Titarenko, A. Hasnaoui, R. Titarenko, and L. Buzuk, "Robust data analysis in innovation project portfolio management," in MATEC Web of Conferences, 2018, vol. 170, p. 1017.

[10] F. Barrios, F. López, L. Argerich, and R. Wachenchauzer, "Variations of the similarity function of textrank for automated summarization," arXiv Prepr. arXiv1602.03606, 2016.

[11] G. Di Fabbrizio, A. Aker, and R. Gaizauskas, "Summarizing Online Reviews Using Aspect Rating Distributions and Language Modeling," IEEE Intell. Syst., vol. 28, no. 3, pp. 28-37, May 2013.

[12] T. J. Kloppenborg, D. Tesch, and R. R. Chinta, "Demographic determinants of project success behaviors," Pulse, vol. 18, 2019.

[13] H. Taherdoost and A. Keshavarzsaleh, "A theoretical review on IT project success/failure factors and evaluating the associated risks," 2018.

[14] C. Symons, "The ROI of project portfolio management tools," Forrester, May, vol. 8, 2009.

[15] D. Lock and R. Wagner, The Handbook of Project Portfolio Management. Routledge, 2018.

[16] R. R. Nelson, "IT project management: Infamous failures, classic mistakes, and best practices.," MIS Q. Exec., vol. 6, no. 2, 2007.

[17] K. Heldman, PMP: project management professional exam study guide. John Wiley \& Sons, 2018.

[18] J. A. Araúzo, J. Pajares, and A. Lopez-Paredes, "Simulating the dynamic scheduling of project portfolios," Simul. Model. Pract. Theory, vol. 18, no. 10, pp. 1428-1441, 2010.

[19] S. Rajegopal, P. McGuin, and J. Waller, Project portfolio management: Leading the corporate vision. Springer, 2007.

[20] P. Andy, "IDC: Expect 175 zettabytes of data worldwide by 2025," 2018. [Online]. Available: https://www.networkworld.com/article/ 3325397/idc-expect-175-zettabytes-of-data-worldwide-by-2025.html. [Accessed: 01-Apr-2019].

[21] Gartner® Inc, "FrontRunners Methodology,” 2018. [Online]. Available: https://www.saimgs.com/imglib/other_pages/FrontRunners/Methodolog yOverview.pdf. [Accessed: 28-Feb-2019].

[22] H. Kerzner, Project management best practices: Achieving global excellence. John Wiley \& Sons, 2018.

[23] C. N. Enoch, Project portfolio management: a model for improved decision making. Business Expert Press, 2019.

[24] L. K. Hansen and P. Svejvig, "Towards rethinking Project portfolio management," 2018.

[25] B. Liu, M. Hu, and J. Cheng, "Opinion observer: analyzing and comparing opinions on the Web," in Proceedings of the 14th international conference on World Wide Web, 2005, pp. 342-351.

[26] R. Řehưřek and P. Sojka, "Software Framework for Topic Modelling with Large Corpora," in Proceedings of the LREC 2010 Workshop on New Challenges for NLP Frameworks, 2010, pp. 45-50.

[27] Q. Mei, X. Ling, M. Wondra, H. Su, and C. Zhai, "Topic sentiment mixture: modeling facets and opinions in weblogs," in Proceedings of the 16th international conference on World Wide Web, 2007, pp. 171180.

[28] D. M. Blei, A. Y. Ng, and M. I. Jordan, "Latent dirichlet allocation," J. Mach. Learn. Res., vol. 3, pp. 993-1022, Mar. 2003. 